Article

\title{
Pyrolysis Combustion Flow Calorimetry Studies on Some Reactively Modified Polymers
}

\section{Svetlana Tretsiakova-McNally and Paul Joseph *}

School of the Built Environment and the Built Environment Research Institute, Ulster University, Newtownabbey BT37 0QB, UK; E-Mail: s.tretsiakova-mcnally@ulster.ac.uk

* Author to whom correspondence should be addressed; E-Mail: p.joseph@ulster.ac.uk; Tel.: +44-28-9036-8755; Fax: +44-28-9036-8726.

Academic Editor: A. Richard Horrocks

Received: 8 December 2014 / Accepted: 24 February 2015 / Published: 2 March 2015

\begin{abstract}
As a part of our continuing work to improve the flame retardance of some chain-growth polymers, by employing a reactive route, we have synthesized several unsaturated compounds containing either phosphorus $(\mathrm{P})$, or both phosphorus $(\mathrm{P})$ and nitrogen $(\mathrm{N})$, bearing groups in different chemical environments. They included: diethyl(acryloyloxymethyl)phosphonate (DEAMP); diethyl(1-acryloyloxyethyl)phosphonate (DE1AEP); diethyl-2-(acryloyloxy)ethyl phosphate (DEAEP); diethyl-2-(metharyloyloxy)ethyl phosphate (DEMEP); acrylic acid-2-(diethoxyphosphorylamino)ethyl ester (ADEPAE); acrylic acid-2-[(diethoxyphosphoryl)methyl amino]ethyl ester (ADEPMAE). Acrylonitrile (AN), methyl methacrylate (MMA) and styrene (S) were free radically copolymerised with the above mentioned comonomers. The recovered polymers were subjected to routine spectroscopic and thermo-gravimetric analyses. In addition, the combustion behaviours of homopolymers as well as the copolymers containing nominal loadings of $\mathrm{P}-$, or $\mathrm{P} / \mathrm{N}-$, groups were, primarily, evaluated using pyrolysis combustion flow calorimetry (PCFC). PCFC has been found to be a very useful screening technique, especially, in establishing the efficacies of the different modifying groups towards flame retarding some base polymeric materials. Values of the heat release capacity (HRC) values normalised to the P contents $(\mathrm{wt} \%$ ) can be considered as useful tool in ranking the various P-containing modifying groups in terms of their efficacies to flame-retard non-halogenated chain-growth polymers considered in the present work.
\end{abstract}


Keywords: chain-growth polymers; chemical modification; flame retardance; pyrolysis combustion flow calorimetry; heat release rate; heat release capacity

\section{Introduction}

A wide range of qualitative, semi-quantitative and quantitative testing techniques are currently available, both at the laboratory scale and for commercial purposes, to evaluate the degradation and combustion behaviours of polymeric materials. They include, but not limited to, techniques such as: thermo-gravimetric analysis (TGA), oxygen bomb calorimetry, limiting oxygen index (LOI) measurements, Underwriters Laboratory 94 (UL-94) tests, cone calorimetry, etc. All of the above mentioned methods have their own advantages as well as limitations. For example, the LOI test determines the propensity of the material to undergo ignition and flaming combustion in a pre-set atmosphere of oxygen and nitrogen, under the influence of a pilot, and thus only reflects a low level flammability attribute of the material under consideration. Furthermore, this method does not provide other valuable information, for instance, the heat release rates. UL-94 test, which is commonly used as an industry standard, can be only considered as a qualitative flammability test method leading to a classification of the materials, and the main outcome of the test will be a pass or fail criteria, even though it can somewhat gauge the propensity of the material to melt and drip. Cone calorimetry, which is widely regarded as the standard method to evaluate the overall fire performance of a material, is based on the oxygen consumption calorimetry principle, and provides valuable parameters like: time to ignition, mass loss rate, heat release rates (peak and average), total heat released, effectiveness of combustion (through $\mathrm{CO} / \mathrm{CO}_{2}$ ratios), smoke obscuration, etc. However, for typical measurements, often carried out in triplicate and under differing heat fluxes, cone calorimetry measurements require relatively high amounts of materials. Ideally, each test specimen should be of about $10 \mathrm{~cm} \times 10 \mathrm{~cm} \times 6 \mathrm{~mm}$ in dimension, thus requiring at least $50 \mathrm{~g}$ of material. In addition, there are also issues with varying sample thicknesses, and associated problems of heat losses which are also related to insulation material used to support the specimens in sample holders, and the actual material of the holder and/or its configurations.

Pyrolysis Combustion Flow Calorimetry (PCFC), also known as micro-scale combustion calorimetry, was shown to be a very valuable small-scale technique for screening flammability of different materials in recent years [1]. We have chosen PCFC for our studies as a rapid screening technique as it only requires a few milligrams of a polymer for testing, and often provides a wealth of combustion-related data. PCFC works on a principle of oxygen depletion calorimetry, relating to Hugget's principle that $1 \mathrm{~kg}$ of consumed oxygen corresponds to $13.1 \mathrm{MJ}$ of released energy for any organic material [2]. At first, a polymeric sample is rapidly heated to a state of controlled pyrolysis in an inert atmosphere of nitrogen (method A: anaerobic conditions) or in a mixture of nitrogen and oxygen (method B: aerobic conditions), followed by a rapid oxidation at high temperatures (i.e., combustion) of the pyrolysate in an excess of oxygen [3]. In the present study, the operational conditions were in conformance with the test method A. This method also is an established ASTM standard for testing flammability characteristics of solid materials [1]. PCFC is capable to measure the following parameters: peak to heat release rate (PHRR); temperature at peak heat release rate $\left(T_{\mathrm{PHRR}}\right)$; total heat 
release (THR); heat release capacity (HRC) and a percentage of the char residues. The values of HRC (i.e., maximum amount of heat released per unit mass per degree temperature) can serve as a reliable indicator of a polymer's flammability [3].

\section{Experimental Section}

\subsection{Materials}

All chemicals, solvents and other laboratory reagents were purchased from the Sigma Aldrich Company (Gillingham, UK), with the exception of acryloyl chloride (supplied by Alfa Aesar, Heysham, UK). The polymerisation inhibitors were removed from acrylonitrile (AN), styrene (S) and methyl methacrylate (MMA) prior use. AN was freed from the 4-methoxyphenol by passing through a column of activated basic alumina, and then it was stored over molecular sieves ( $4 \AA$ type) at $5{ }^{\circ} \mathrm{C}$. MMA, containing 10-100 ppm of monomethyl ether hydroquinone as an inhibitor, was freed from it by passing through an inhibitor removal pre-packed column and the monomer was stored over molecular sieves ( $4 \AA$ type) in the dark at $5{ }^{\circ} \mathrm{C}$. S, stabilized with $10-15 \mathrm{ppm}$ of 4-tert-butylcatechol, was purified with the aid of a proprietary inhibitor removal column, and the monomer was stored in a sealed vessel below $0{ }^{\circ} \mathrm{C}$. 2,2'-azobisisobutyronitrile (AIBN), which was used as a radical initiator, was purified by recrystallization from methanol. Other reagents and solvents were purified, if necessary, in line with standard literature procedures [4].

\section{2. $P$ - and $P$-/N-Containing Comonomers}

Syntheses of phosphorus- and phosphorus and nitrogen-containing unsaturated compounds were carried out following literature precedents [5-7]. The P-containing monomers prepared for this study included: two acrylic phosphonates: diethyl(acryloyloxymethyl)phosphonate (DEAMP) $(R=\mathrm{H}$, structure 1, Figure 1) and diethyl(1-acryloyloxyethyl)phosphonate (DE1AEP) $\left(R=\mathrm{CH}_{3}-\right.$, structure 1, Figure 1); acrylic phosphate: diethyl-2-(acryloyloxy)ethyl phosphate (DEAEP) $(R=\mathrm{H}$, structure 2, Figure 1), and a methacrylic phosphate: diethyl-2-(metharyloyloxy)ethyl phosphate (DEMEP) $\left(R=\mathrm{CH}_{3}-\right.$, structure 2, Figure 1). In addition, we have synthesized two phosphorylamino esters containing both $\mathrm{P}$ and $\mathrm{N}$ atoms: acrylic acid-2-(diethoxyphosphorylamino)ethyl ester (ADEPAE) $(R=\mathrm{H}$, structure 3, Figure 1) and acrylic acid-2-[(diethoxyphosphoryl)methyl amino]ethyl ester (ADEPMAE) $\left(R=\mathrm{CH}_{3}-\right.$, structure 3, Figure 1). The following criteria were primarily used for the choice of the comonomers: low cost of the reagents required for the laboratory preparation; low toxicity of the reactants and products; appreciable polymerisability of these comonomers with AN, MMA and S; ease of syntheses coupled with appreciable final yields; relatively high hydrolytic stability of the monomers. The chemical structures and the degrees of purities of theses comonomers, as well as their precursors, were evaluated using both GC/MS analyses and NMR $\left({ }^{1} \mathrm{H}\right.$ and $\left.{ }^{31} \mathrm{P}\right)$ spectroscopy. The characteristics of the NMR spectra for all six comonomers were in agreement with previously published data [7-9]. P- and $\mathrm{P}-/ \mathrm{N}$-containing comonomers were produced with typical yields ranging between 68 and $95 \mathrm{wt} \%$. 


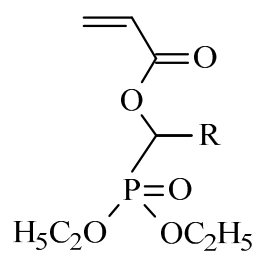

(1)

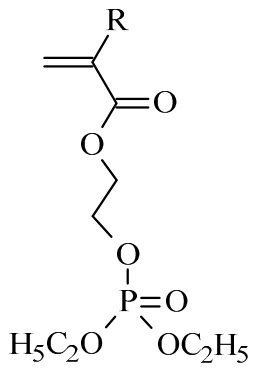

(2)

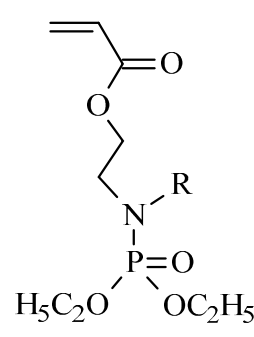

(3)

$\mathrm{R}=\mathrm{H}$ or $\mathrm{CH}_{3}$

Figure 1. Structures of P- or P-/N-containing comonomers: (1) diethyl(acryloyloxymethyl) phosphonate (DEAMP), if $\mathrm{R}=\mathrm{H}$ or diethyl(1-acryloyloxyethyl)phosphonate (DE1AEP), if $R=\mathrm{CH}_{3}$; (2) diethyl-2-(acryloyloxy)ethyl phosphate (DEAEP), if $R=\mathrm{H}$ or diethyl-2-(methacryloxy)ethyl phosphate (DEMEP), if $R=\mathrm{CH}_{3}$; (3) acrylic acid-2(diethoxyphosphorylamino)ethyl ester (ADEPAE), if $R=\mathrm{H}$ or acrylic acid-2[(diethoxyphosphoryl)methyl amino] ethyl ester (ADEPMAE), if $R=\mathrm{CH}_{3}$.

\subsection{Syntheses of Homo- and Co-polymers}

\subsubsection{Preparation of AN-Based Polymers}

Homo- and co-polymerisation reactions of AN with DEAMP, DE1AEP, DEAEP, ADEPMAE and ADEPAE were carried out, in aqueous slurries using sodium metabisulfite and ammonium persulfate as a redox initiator pair, following the method previously reported by us [10]. The isolated polymers were washed several times with deionised water to remove traces on unreacted monomer(s), and then were dried at $c a .60{ }^{\circ} \mathrm{C}$ in a vacuum oven to constant weights. The preparative data for syntheses of homopolymer polyacrylonitrile (PAN) and AN-based copolymers is shown in Table 1.

Table 1. Preparative data for acrylonitrile (AN)-based polymers.

\begin{tabular}{cccccccc}
\hline Comonomer & $\begin{array}{c}\text { AN } \\
\left(\mathbf{c m}^{\mathbf{3}}\right)\end{array}$ & $\begin{array}{c}\text { Comonomer } \\
\mathbf{( g )}\end{array}$ & $\begin{array}{c}\text { AN/comonomer } \\
\text { ratio in the feed } \\
(\mathbf{m o l e} \text { fraction) }\end{array}$ & $\begin{array}{c}\text { Sodium } \\
\text { metabisulfite } \\
(\mathbf{g})\end{array}$ & $\begin{array}{c}\text { Ammonium } \\
\text { persulfate } \\
(\mathbf{g})\end{array}$ & $\begin{array}{c}\text { Water } \\
\left(\mathbf{c m}^{\mathbf{3}}\right)\end{array}$ & $\begin{array}{c}\text { Yield } \\
(\mathbf{w t} \%)\end{array}$ \\
\hline- & 13.00 & - & $1.00 / 0.00$ & 1.00 & 0.35 & 290 & 88 \\
DEAMP & 11.84 & 4.44 & $0.90 / 0.10$ & 1.00 & 0.35 & 290 & 86 \\
DE1AEP & 11.80 & 4.76 & $0.90 / 0.10$ & 1.00 & 0.35 & 290 & 80 \\
DEAEP & 11.80 & 4.90 & $0.90 / 0.10$ & 1.00 & 0.35 & 290 & 82 \\
ADEPMAE & 8.10 & 3.31 & $0.92 / 0.08$ & 1.00 & 0.35 & 290 & 69 \\
ADEPAE & 10.00 & 3.02 & $0.90 / 0.10$ & 1.00 & 0.35 & 290 & 55 \\
\hline
\end{tabular}

\subsubsection{Preparation of MMA-Based Polymers}

Homo- and co-polymers of MMA were prepared by free-radical polymerisations of MMA and corresponding amount of comonomer (DEAMP, DE1AEP, DEAEP, DEMEP, ADEPMAE and ADEPAE) in toluene solution, using AIBN as the initiator, under an argon atmosphere. The required volume of MMA, or a mixture of MMA and a relevant comonomer, was first placed in a three-necked round 
bottomed flask, fitted with magnetic stirrer, a water condenser and a bubbler, and containing dry toluene which was initially flushed with argon at room temperature,. The mixture was stirred for $c a$. $30 \mathrm{~min}$ and gradually heated to $60{ }^{\circ} \mathrm{C}$ with argon bubbling through it. Once the temperature reached $60{ }^{\circ} \mathrm{C}$ the initiator, AIBN (concentration was $c a .2 \mathrm{~g} / \mathrm{L}$ in all cases), was added to the reaction mixture. The argon inlet was withdrawn from the reaction mixture, and the polymerisation was allowed to proceed for 5-6 h under a blanket of argon. The viscosity of the reaction mixture was closely monitored during this period to gauge the progress of the polymerisation reaction. The resulting homoand co-polymers were recovered by the precipitation of reaction mixture into a five-fold excess of heptane. The white powdery materials were collected by filtration, washed several times with heptane to remove unreacted comonomer(s), and then dried to constant weights in a vacuum oven at $50-55{ }^{\circ} \mathrm{C}$ before further examinations. The preparative data for MMA-based polymers is summarised in Table 2.

Table 2. Preparative data for methyl methacrylate (MMA)-based polymers.

\begin{tabular}{cccccccc}
\hline Comonomer & $\begin{array}{c}\text { MMA } \\
\left(\mathbf{c m}^{\mathbf{3}}\right)\end{array}$ & $\begin{array}{c}\text { Comonomer } \\
\mathbf{( g )}\end{array}$ & $\begin{array}{c}\text { MMA/comonomer } \\
\text { ratio in the feed } \\
(\mathbf{m o l e} \text { fraction) }\end{array}$ & $\begin{array}{c}\text { AIBN } \\
(\mathbf{m g})\end{array}$ & $\begin{array}{c}\text { Toluene } \\
\left(\mathbf{c m}^{\mathbf{3}}\right)\end{array}$ & $\begin{array}{c}\text { Heptane } \\
\left(\mathbf{c m}^{\mathbf{3}}\right)\end{array}$ & $\begin{array}{c}\text { Yield } \\
(\mathbf{g})\end{array}$ \\
\hline- & 12.0 & - & $1.00 / 0.00$ & 96 & 36 & 300 & 5.50 \\
DEAMP & 9.6 & 2.22 & $0.90 / 0.10$ & 74 & 25 & 300 & 2.90 \\
DE1AEP & 9.6 & 2.36 & $0.90 / 0.10$ & 74 & 24 & 300 & 2.63 \\
DEAEP & 9.6 & 2.52 & $0.90 / 0.10$ & 76 & 25 & 300 & 3.21 \\
DEMEP & 9.6 & 2.66 & $0.90 / 0.10$ & 76 & 25 & 300 & 1.94 \\
ADEPMAE & 9.6 & 2.65 & $0.90 / 0.10$ & 74 & 24 & 300 & 5.41 \\
ADEPAE & 9.6 & 2.51 & $0.90 / 0.10$ & 74 & 24 & 300 & 2.80 \\
\hline
\end{tabular}

\subsubsection{Preparation of S-Based Polymers}

The procedures for syntheses of homo- and co-polymers of S were similar to those described for MMA-based polymers (Section 2.3.2). However, there were two main differences: (1) the use of methanol as a non-solvent to recover prepared polymers; (2) the duration of homo-/co- polymerisations was about $16 \mathrm{~h}$ to achieve the required levels of conversion.

The resulting polymers, in the form of white powders, after washing, were dried to constant weights in a vacuum oven at about $50-55^{\circ} \mathrm{C}$. The preparative data for syntheses of $\mathrm{S}$ and its copolymers are provided in Table 3.

Table 3. Preparative data for styrene (S)-based polymers.

\begin{tabular}{cccccccc}
\hline Comonomer & $\mathbf{S}\left(\mathbf{c m}^{\mathbf{3}}\right)$ & Comonomer $\mathbf{( g )}$ & $\begin{array}{c}\text { S/comonomer } \\
\text { ratio in the feed } \\
(\mathbf{m o l e} \text { fraction) }\end{array}$ & $\begin{array}{c}\text { AIBN } \\
(\mathbf{m g})\end{array}$ & $\begin{array}{c}\text { Toluene } \\
\left(\mathbf{c m}^{\mathbf{3}}\right)\end{array}$ & $\begin{array}{c}\text { Methanol } \\
\left(\mathbf{c m}^{\mathbf{3}}\right)\end{array}$ & $\begin{array}{c}\text { Yield } \\
(\mathbf{g})\end{array}$ \\
\hline- & 12.0 & - & $1.00 / 0.00$ & 104 & 40 & 400 & 4.50 \\
DEAMP & 10.3 & 2.02 & $0.90 / 0.10$ & 80 & 30 & 300 & 3.00 \\
DE1AEP & 10.3 & 2.34 & $0.90 / 0.10$ & 100 & 40 & 400 & 4.06 \\
DEAEP & 10.3 & 2.52 & $0.90 / 0.10$ & 100 & 40 & 500 & 4.30 \\
DEMEP & 10.3 & 2.68 & $0.90 / 0.10$ & 80 & 30 & 300 & 3.99 \\
ADEPMAE & 10.3 & 2.65 & $0.90 / 0.10$ & 80 & 30 & 400 & 3.68 \\
ADEPAE & 10.3 & 2.51 & $0.90 / 0.10$ & 100 & 40 & 500 & 2.82 \\
\hline
\end{tabular}




\subsection{Characterization Techniques}

For GC-MS (Gas Chromatography-Mass Spectrometry) runs, the chromatograms and the corresponding mass spectra were obtained using Agilent 6890N gas chromatograph coupled with Agilent 5973N mass spectrometer (Agilent Technologies, Manchester, UK). Agilent Technologies HP-Ultra 2 $(25 \mathrm{~m} \times 0.2 \mathrm{~mm} \times 0.33 \mu \mathrm{m})$ column was used for the GC runs. Chemical ionization was carried out using methane with the mass spectrometer scanning the mass range of 100-600 amu.

${ }^{1} \mathrm{H}$ and ${ }^{31} \mathrm{P}$ NMR (Nuclear Magnetic Resonance) spectra of starting materials, monomers and polymers were recorded in deuterated solvents $\left(\mathrm{CDCl}_{3}\right.$ or $\mathrm{d}_{6}$-DMSO (dimethyl sulfoxide)) on Bruker spectrometers, operating at either $400 \mathrm{MHz}$ for protons, or at $500 \mathrm{MHz}$ for phosphorus, under ambient probe conditions. The spectra were processed using WIN-NMR software (Bruker, Coventry, UK) after being calibrated using the residual proton signals or the main carbon signals arising from the solvents. For ${ }^{31} \mathrm{P}$ NMR, $85 \%$ orthophosphoric acid was used as an external calibrant.

Depending of the solubility of polymeric products obtained, they were dissolved either in d6-DMSO or in $\mathrm{CDCl}_{3}$, for the spectral measurements. The ${ }^{1} \mathrm{H}$ NMR spectrum of each polymer was examined firstly to ensure the absence of the residual monomer in the final polymeric product, and secondly to calculate the degree of incorporation of $\mathrm{P}$ - or $\mathrm{P}-\mathrm{N}$ - containing comonomer. The general chemical compositions of the copolymers can be represented as follows:

$$
[\mathrm{M}]_{x}-[\operatorname{co}-\mathrm{M}]_{(1-x)}
$$

where $[\mathrm{M}]$ are monomeric units of either AN, or MMA, or S, and $x$ is their mole fraction in a copolymer; [co-M] are P- or P/N-containing comonomeric units (DEAMP, DE1AEP, DEAEP, DEMEP, ADEPMAE, or ADEPAE) and $(1-x)$ is their mole fraction within a copolymer. The mole fractions of units [M] and $[\mathrm{co}-\mathrm{M}]$ in the modified polymers were obtained by comparing the integrated areas of appropriately assigned signals. Then the content of phosphorus ( $\mathrm{wt} \%)$ in a copolymer was calculated from the following equation:

$$
\% P=\frac{(1-x) \cdot 31 \cdot 100 \%}{x \cdot \mathrm{M}_{[M]}+(1-x) \cdot \mathrm{M}_{[c o-M]}}
$$

where $\mathrm{M}_{[M]}$ is a molecular mass of a monomeric unit $\mathrm{M} ; \mathrm{M}_{[c o-M]}$ is a molecular mass of comonomeric unit, co-M.

Pyrolysis Combustion Flow Calorimetric (PCFC) measurements were performed using a Fire Testing Technology Ltd. (Gosport, UK) micro-scale combustion calorimeter. The description of this method including the operating parameters is published in detail elsewhere [3]. The following PCFC parameters were measured: peak heat release rate (PHRR); temperature at peak heat release rate

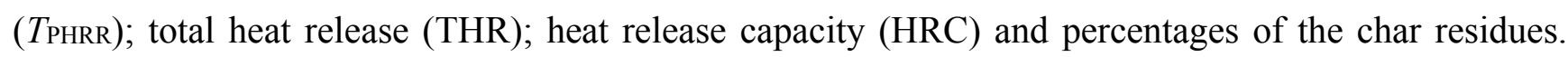
The instrument also recorded plots of the Heat Release Rate (HRR) versus temperature (or versus time). For each run, accurately weighed (ca. $5 \mathrm{mg}$ ) and finely powdered sample was first heated to about $900{ }^{\circ} \mathrm{C}$ at a heating rate of $0.9{ }^{\circ} \mathrm{C} / \mathrm{s}$, in a stream of nitrogen flowing at a rate of $80 \mathrm{~cm}^{3} / \mathrm{min}$. The volatile thermal degradation products, thus obtained, were then mixed with a stream of pure oxygen (at a flow rate of $20 \mathrm{~cm}^{3} / \mathrm{min}$ ) prior to entering a combustion chamber maintained at $900{ }^{\circ} \mathrm{C}$. Each sample was run in triplicate. The data obtained and presented in this paper are average values of 
three measurements. Generally, the variation among the values of PHRR, TPHRR, THR, and HRC measurements did not exceed $6 \%$, while those for char residues reached up to $14.8 \%$. For example, Table 4 provides the average values, standard deviations and coefficients of variation (\%) for the control sample of polymethyl methacrylate (PMMA).

Table 4. The standard deviation and the coefficient of variation of the pyrolysis combustion flow calorimetry (PCFC) measurements for unmodified polymethyl methacrylate (PMMA).

\begin{tabular}{lcccccc}
\hline Sample & Run/calculation & PHRR $(\mathbf{W} / \mathbf{g})$ & T $_{\text {PHRR }}\left({ }^{\circ} \mathbf{C}\right)$ & THR $(\mathbf{k J} / \mathbf{g})$ & HRC $(\mathbf{J} / \mathbf{g} \cdot \mathbf{K})$ & Char residue $(\mathbf{w t} \%)$ \\
\hline PMMA & 1 & 306 & 375 & 26.2 & 337 & 0.9 \\
PMMA & 2 & 307 & 378 & 29.2 & 337 & 1.0 \\
PMMA & 3 & 310 & 383 & 28.1 & 341 & 1.2 \\
PMMA & Average & 307.7 & 378.7 & 27.8 & 337.9 & 1.03 \\
PMMA & Standard deviation & 2.08 & 4.04 & 1.52 & 2.31 & 0.15 \\
PMMA & Coefficient of variation, $\%$ & 0.7 & 1.1 & 5.5 & 0.7 & 14.8 \\
\hline
\end{tabular}

The thermo-gravimetric analyses (TGA) were performed with the aid of a Mettler Toledo TGA/SDTA851 ${ }^{\mathrm{e}}$ instrument. The TGA were carried out on $c a .10 \mathrm{mg}$ of samples, at a heating rate of $10{ }^{\circ} \mathrm{C} / \mathrm{min}$ in atmosphere of nitrogen at a flow rate of $50 \mathrm{~cm}^{3} / \mathrm{min}$, from 30 to $700{ }^{\circ} \mathrm{C}$. Optionally, the TGA runs were also repeated in oxygen and in air.

\section{Results and Discussion}

The aqueous slurry route was found to be a quite facile method for the preparation of polymeric products from AN, which provided typical yields of homo- and co-polymers between 55 and $88 \mathrm{wt} \%$ (Table 1). It was previously shown that chemical modification of PAN through the incorporation of ADEPMAE groups significantly increased its flame retardance [10]. In this case, we have also previously noticed strong correlation between the char residues obtained in nitrogen, air and in oxygen, and the $\mathrm{wt} \%$ of $\mathrm{P}$ in $\mathrm{AN}-$ based polymers containing $\mathrm{P} / \mathrm{N}$-moieties. In addition, corresponding increases in LOI values coupled to decreases in heats of combustion were also found for these materials (see reference [10] for detailed data). The above factors show that even with nominal amounts of $\mathrm{P}$, there were substantial enhancements in flame retardance of the modified systems. Furthermore, as is expected, above certain levels ( $c a .3 \mathrm{wt} \%$ ) of $\mathrm{P}$ incorporation there were indications of a diminished returns. It was also evident from the measured PCFC parameters that this effect is most pronounced for copolymers containing 1.18-1.41 wt\% of $\mathrm{P}$ (corresponding mole fractions of ADEPMAE in the reaction feed ranged from 0.06 to 0.10 ).

In the current study, we intended to achieve similar contents of modifying groups (i.e., mole fractions of $\mathrm{P}$ - or $\mathrm{P} / \mathrm{N}$-containing monomeric units) and comparable $\mathrm{P}$ loadings. Even though we have kept the same amount of the comonomer [co-M] in the feed (0.08-0.10 mole fraction), constant temperature $\left(40^{\circ} \mathrm{C}\right)$ and almost the same duration of polymerisation $(16 \mathrm{~h})$ with the same amount of the redox pair for all reactions carried out, the yields and the compositions of resultant copolymers did vary (Table 5). The P contents in the prepared AN-based copolymers were within 1.0-1.2 wt\% range with the exception of poly(AN-co-ADEPAE), in which the level of P reached $1.9 \mathrm{wt} \%$. This can be explained by different chemical environments for the penta-valent P-atom, which is expected to 
influence their reactivity ratios with AN. All the final polymeric products were white, powdery solids without any signs of discoloration. This indicates the absence of unwanted side reactions, such as intramolecular cyclization of nitrile groups in AN-based polymers, which could have led to extended conjugation and light absorption.

Table 5. PCFC parameters for AN-based polymeric systems.

\begin{tabular}{ccccccc}
\hline Parameter & PAN & $\begin{array}{c}\text { Poly(AN-co- } \\
\text { DEAMP) }\end{array}$ & $\begin{array}{c}\text { Poly(AN-co- } \\
\text { DE1AEP) }\end{array}$ & $\begin{array}{c}\text { Poly(AN-co- } \\
\text { DEAEP) }\end{array}$ & $\begin{array}{c}\text { Poly(AN-co- } \\
\text { ADEPMAE) }\end{array}$ & $\begin{array}{c}\text { Poly(AN-co- } \\
\text { ADEPAE) }\end{array}$ \\
\hline$[\mathrm{M}] /[\mathrm{co}-\mathrm{M}]($ mole fraction) & $1.0 / 0.0$ & $0.982 / 0.018$ & $0.980 / 0.020$ & $0.978 / 0.022$ & $0.978 / 0.022$ & $0.963 / 0.037$ \\
P content (wt\%) & 0 & 1.0 & 1.1 & 1.2 & 1.2 & 1.9 \\
$T_{\text {PHRR }}\left({ }^{\circ} \mathrm{C}\right)$ & 289 & 429 & 432 & 426 & 426 & 410 \\
PHRR (W/g) & 183 & 112 & 122 & 114 & 85 & 106 \\
PHRR (\% reduction) & 0 & 39 & 33 & 38 & 54 & 42 \\
THR (kJ/g) & 14.9 & 17.5 & 16.8 & 14.3 & 8.9 & 15.9 \\
HRC $(\mathrm{J} / \mathrm{g} \cdot \mathrm{K})$ & 201 & 122 & 132 & 124 & 86 & 115 \\
PCFC char yield $(\mathrm{wt} \%)$ & 45.6 & 58.4 & 55.8 & 58.2 & 53.6 & 55.3 \\
\hline
\end{tabular}

Figure 2 represents plots of HRR versus temperature for PAN and for selected copolymers containing from 1.0 to $1.2 \mathrm{wt} \%$ of $\mathrm{P}$ as pendant groups (DEAMP, or DEAEP, or ADEPMAE). A single prominent heat release rate peak (with a maximum at $289^{\circ} \mathrm{C}$ ) was observed for the unmodified PAN. As it shown on Figure 2, the incorporation even at a very nominal level of P into PAN chains as pendant groups drastically changed the general profiles of HRR curves. Indeed, two peaks were observed for copolymers: first (either small or very narrow) peak at $280-290{ }^{\circ} \mathrm{C}$ and the second one (higher and very broad peak) shifted to higher temperatures with the maximum at 390-430 ${ }^{\circ} \mathrm{C}$. At the same time for the copolymers the heights of peaks as well as the areas under the curves were significantly reduced compared to PAN. Figure 2 also shows that thermal degradation of copolymers began $20-50{ }^{\circ} \mathrm{C}$ earlier compared to PAN. The same trends were observed during TGA runs on the specimens tested in nitrogen atmosphere (data not reported here). In the case of the copolymers, values of TPHRR for heat releases associated with earlier induction of thermal degradation (first peaks) were not reported in Table 5.

The data summarised in Table 5 indicates that the reactive modification of PAN with P- or $\mathrm{P}-\mathrm{N}$-containing groups leads to the reduction of PHRR values by a factor of 1.5 to 2.2. The lowest value of PHRR was recorded for the copolymer bearing ADEPMAE groups $(85 \mathrm{~W} / \mathrm{g})$. The maximum value of heat release rate divided by the heating rate of PCFC test gives a value of heat release capacity (HRC), which can be used as a reliable indicator of a polymer's flammability [2]. It is shown here that the presence $\mathrm{P}$ - or $\mathrm{P} / \mathrm{N}$-containing comonomeric units in polymeric chains lead to a decrease of HRC values, thus possibly increasing the flame retardance. The copolymer containing $1.2 \mathrm{wt} \%$ of $\mathrm{P}$ and with a synergistic element, such as nitrogen (i.e., ADEPMAE monomeric unit), was characterised by the lowest value of HRC among all AN-based copolymers obtained in the present study. Overall, the modification of PAN with nominal amounts of $\mathrm{P}$ - or P-/N- containing comonomers reduced its HRC by almost $50 \%$. The THR is the amount of heat released throughout the decomposition, in a PCFC run, and can be indicative of the total amount of fuel generated. Poly(AN-co-ADEMPAE) had the lowest value of THR $8.9 \mathrm{~kJ} / \mathrm{g}$, which is 1.7 times lower than that of PAN (Table 5). 


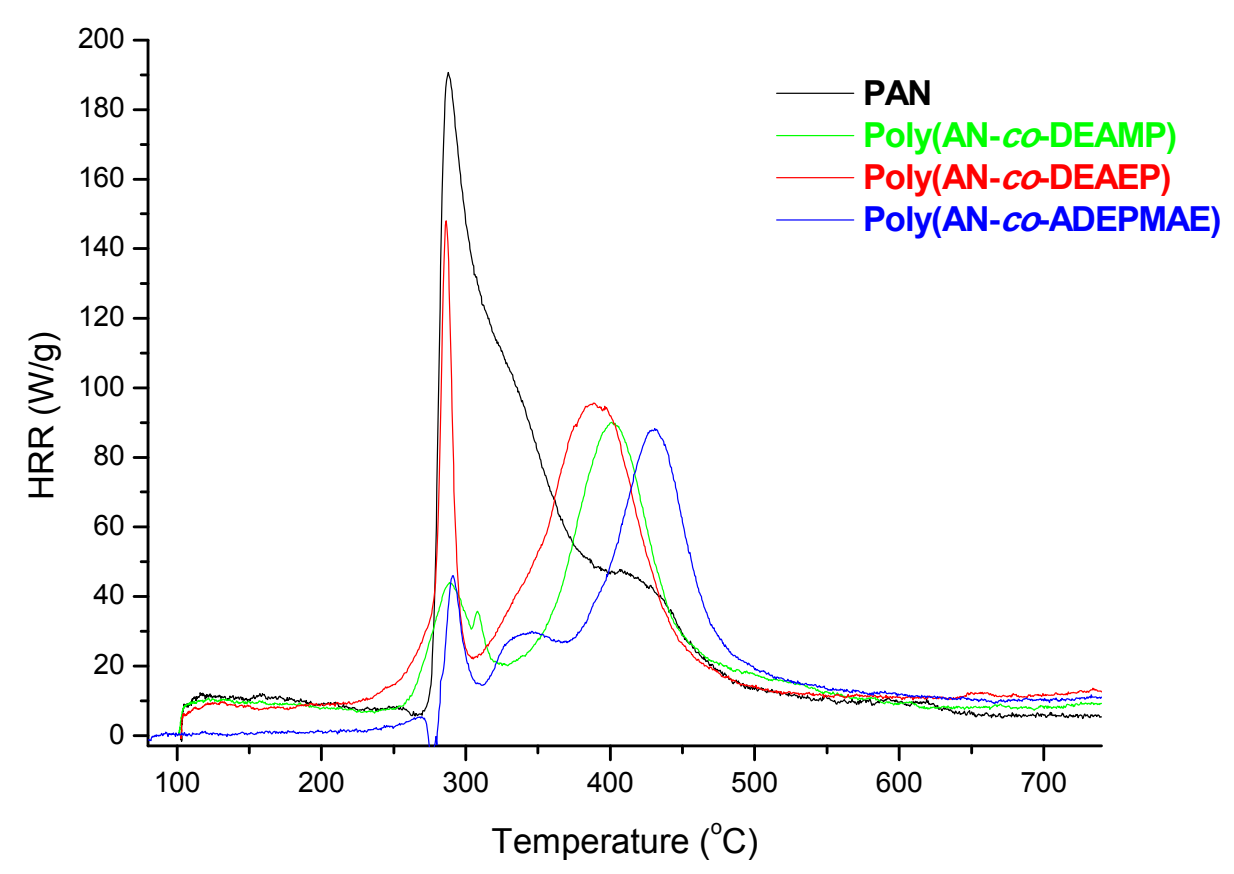

Figure 2. Plots of heat release rates versus temperature for PAN, poly(AN-co-DEAMP), poly(AN-co-DEAEP), and poly(AN-co-ADEPMAE).

Table 5 also shows the char yields obtained in PCFC tests for PAN and for its copolymers. It should be also noted here that the percentages of residues left in TGA experiments, under nitrogen, also correlated well with the corresponding values recorded in PCFC. Even without the incorporation of phosphorus moieties, PAN produces appreciable amount of char residue (45.6 $\mathrm{wt} \%$ in PCFC). The enhanced char production tendency of PAN can be explained by the processes of intramolecular cyclization of $-\mathrm{C} \equiv \mathrm{N}$ side groups occurring, especially predominant, at lower heating rates [11]. As a result, polycyclic structures are formed and nitrogen atoms become 'locked' onto conjugated polyene sequences leading to char precursors, as is shown in Figure 3 below.<smiles>CC1=NC2=NC3=NC4=NC(C)C(C)CC4CC3CC2CC1C</smiles>

Figure 3. Cyclic structures formed due to intramolecular cyclization in PAN.

In case of AN-based copolymers the amounts of char produced were higher than for PAN by about $8-13 \mathrm{wt} \%$. This is due to the fact that phosphorus acid species produced during early stages of the thermal degradation of copolymers had acted as nucleophilic centres, thus promoting intramolecular cyclization reactions $[7,11]$. It is very likely that the mechanism of flame retardation in the modified AN-based polymers involves a significant condensed phase activity initiated by the precursors formed during early thermal cracking (as evident also from the TGA thermograms) of the P- or P-/N-containing side groups $[10,11]$. The condensed phase activity of the $\mathrm{P}$ - or $\mathrm{P} / \mathrm{N}$-containing modifying groups in PAN chains, in enhancing the extent of intramolecular cyclization, thus leads to increased production of char. In addition, vapour-phase inhibitory effects of P-containing moieties (mainly oxides of phosphorus such as $\mathrm{PO}, \mathrm{PO}_{2}, \mathrm{P}_{2} \mathrm{O}_{4}$, etc.) also need to be considered [11]. 
Figure 4 shows a scatter plot of HRC values normalized to the content of $\mathrm{P}$ versus the amount of char produced in PCFC tests, which might be a useful way of ranking the comonomers in their efficiency to improve flame retardance of PAN. Although the incorporation of phosphorylamino ester groups (ADEMPAE and ADEPAE) is quite effective in reducing HRC values, the amounts of corresponding char produced from these copolymers are lower than those for the copolymers containing acrylic phosphate (DEAEP) or acrylic phosphonate (DE1AEP or DEAMP) monomeric units. On the contrary, modification of PAN with DEAEP, DE1AEP and DEAMP enhances the char formation processes but seems to do not concomitantly reduce the HRC values. These inconsistencies might arise from the varying relative degrees of efficacies, shown by the modifying groups with different chemical environments, acting in the condensed and in vapour phases.

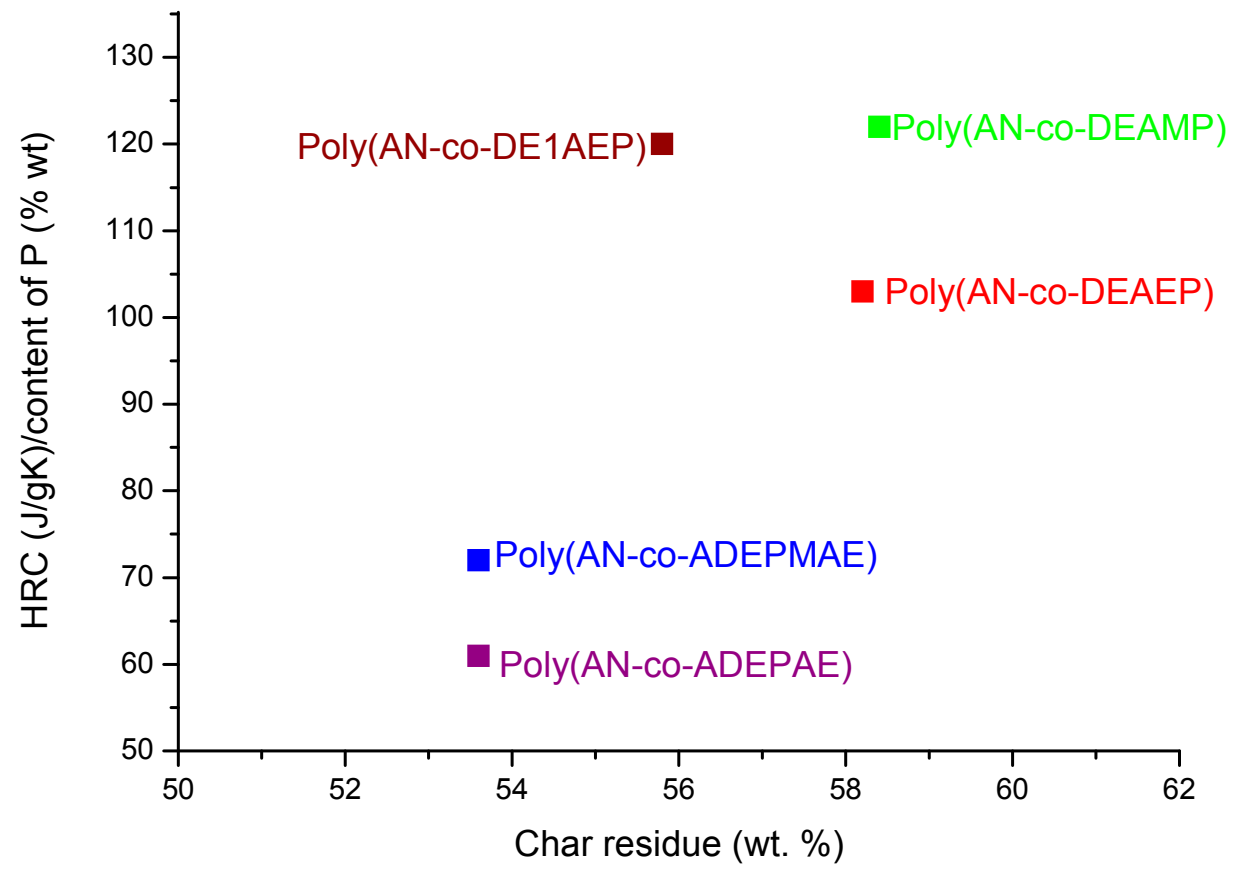

Figure 4. Ratio of HRC to content of P versus PCFC char residue for copolymers of AN.

In the case of MMA- and S-based systems, generally, relatively lower yields of final recovered products were obtained. Furthermore, as with AN-based polymers, it was difficult to achieve the similar mole fractions of modifying groups for all copolymers, and thus the level of $\mathrm{P}$ incorporation varied in the polymeric products obtained (Tables 6 and 7).

In Figure 5, a plot of heat release rates versus temperature for PMMA and for the copolymers with closer levels of P loadings, from 3.2 to $3.4 \mathrm{wt} \%$ are shown. As it is evident from this figure, the incorporation of P- or P-/N-containing groups substantially lowers the PHRR of copolymers as compared to homopolymer. This effect was most pronounced for poly(MMA-co-ADEPAE) that contained both $\mathrm{P}$ and $\mathrm{N}$ atoms in the pendent groups. The temperatures to PHRR associated with the main degradation steps of this copolymer were shifted to higher temperatures compared to PMMA. The PCFC parameters for MMA-based polymeric systems are collated in Table 6. These data also confirm that the highest level of flame retardation for MMA-based copolymers was achieved by incorporating ADEPAE units (0.126 mole fractions, $3.3 \mathrm{wt} \% \mathrm{P})$ into MMA chains. Indeed, the presence of $\mathrm{P}$ and $\mathrm{N}$-containing moieties had led to the decrease, compared to the values recorded for 
PMMA, of: PHRR values by $141 \mathrm{~W} / \mathrm{g}$; THR values by $9.2 \mathrm{~kJ} / \mathrm{g}$; HRC values by $158 \mathrm{~J} / \mathrm{g} \cdot \mathrm{K}$. At the same time, the amount of residue of poly(MMA-co-ADEPAE) was significantly greater $(16.2 \mathrm{wt} \%$ of the initial sample weight) than for unmodified PMMA (0.7 wt\%).

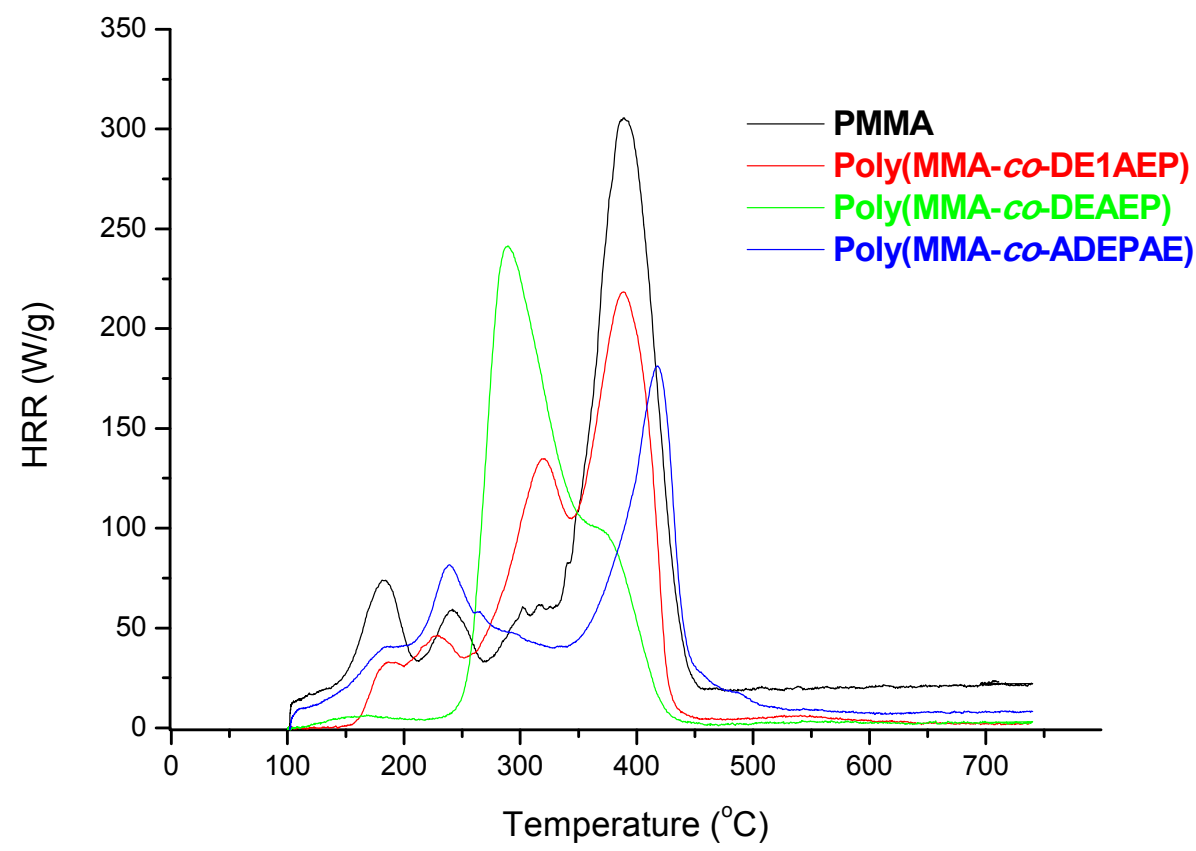

Figure 5. Plots of HRR versus temperature for PMMA, poly(MMA-co-DE1AEP), poly(MMA-co-DEAEP), and poly(MMA-co-ADEPAE).

Table 6. PCFC parameters for homo- and co-polymers of MMA.

\begin{tabular}{|c|c|c|c|c|c|c|c|}
\hline Parameter & PMMA & $\begin{array}{l}\text { Poly(MMA- } \\
\text { co-DEAMP) }\end{array}$ & $\begin{array}{l}\text { Poly(MMA- } \\
\text { co-DE1AEP) }\end{array}$ & $\begin{array}{l}\text { Poly(MMA- } \\
\text { co-DEAEP) }\end{array}$ & $\begin{array}{l}\text { Poly(MMA- } \\
\text { co-DEMEP) }\end{array}$ & $\begin{array}{c}\text { Poly(MMA- } \\
\text { co-ADEPMAE) }\end{array}$ & $\begin{array}{l}\text { Poly(MMA- } \\
\text { co-ADEPAE) }\end{array}$ \\
\hline $\begin{array}{c}{[\mathrm{M}] /[\text { co-M }]} \\
\text { (mole fraction) }\end{array}$ & 0 & $0.890 / 0.110$ & $0.880 / 0.120$ & $0.870 / 0.130$ & $0.900 / 0.100$ & $0.934 / 0.066$ & $0.874 / 0.126$ \\
\hline $\begin{array}{c}\text { P content } \\
(\mathrm{wt} \%)\end{array}$ & 0 & 3.0 & 3.2 & 3.4 & 2.4 & 1.8 & 3.3 \\
\hline$T_{\text {PHRR }}\left({ }^{\circ} \mathrm{C}\right)$ & 379 & 413 & 394 & 291 & 395 & 411 & 414 \\
\hline PHRR (W/g) & 308 & 227 & 206 & 242 & 235 & 234 & 167 \\
\hline $\begin{array}{c}\text { PHRR } \\
\text { (\% reduction) }\end{array}$ & 0 & 26 & 33 & 21 & 24 & 24 & 46 \\
\hline THR $(\mathrm{kJ} / \mathrm{g})$ & 31.1 & 27.4 & 22.9 & 23.1 & 31.8 & 25.7 & 21.9 \\
\hline $\operatorname{HRC}(\mathrm{J} / \mathrm{g} \cdot \mathrm{K})$ & 338 & 246 & 226 & 265 & 256 & 257 & 180 \\
\hline $\begin{array}{c}\text { PCFC char yield } \\
(\mathrm{wt} \%)\end{array}$ & 0.7 & 2.0 & 1.0 & 1.3 & 3.7 & 0.8 & 16.2 \\
\hline
\end{tabular}

Generally, the values of PHRR, THR and HRC of copolymers containing only $\mathrm{P}$ atoms (i.e., as phosphonate groups, DEAMP and DE1AEP, or as phosphate groups, DEAEP and DEMEP) were lower than for unmodified PMMA, but higher compared to the copolymer containing both $\mathrm{P}$ and $\mathrm{N}$ atoms (ADEPAE). The ranking exercise demonstrated that the most efficient reacting comonomer is ADEPAE (Figure 6). Furthermore, DE1AEP appears to be the most efficient, in terms of reducing the flammability, among the copolymers containing $\mathrm{P}$ atom only. 


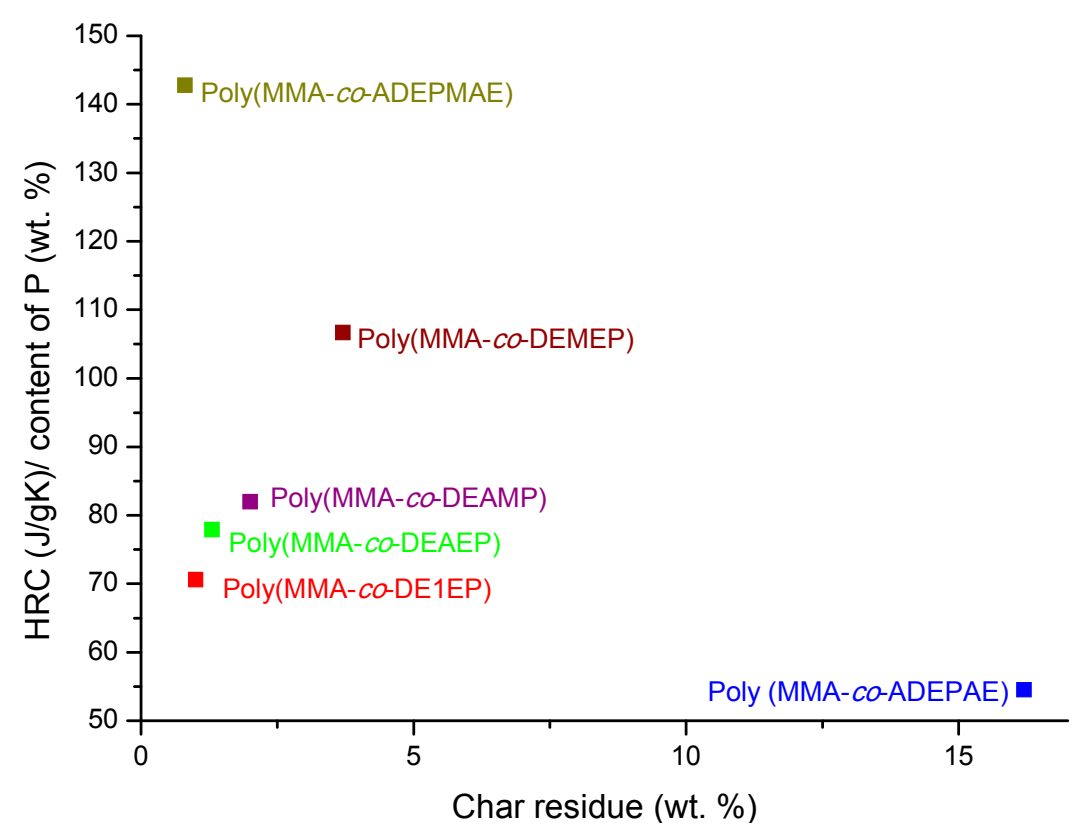

Figure 6. Ratio of HRC to the content of $\mathrm{P}$ versus $\mathrm{PCFC}$ char residue for copolymers of MMA.

As it follows from Table 7 and Figure 7, for S-based polymeric systems the general trends were similar to those of the systems based on PMMA especially, in terms of HRR-temperature profiles and char production tendencies. As opposed to the unmodified counterpart, reactive modification of PS with DEMEP comonomeric units ( 0.127 mole fraction, $3.2 \mathrm{wt} \%$ of $\mathrm{P})$ had led to the most drastic decrease of PHRR and HRC values, whilst the amounts of residue registered by PCFC increased by three-fold. It is interesting to note that copolymerization reaction with $\mathrm{P}$ - and $\mathrm{N}$-containing comonomer (e.g., with ADEPAE) achieved the lowest level of P incorporation (only $0.2 \mathrm{wt} \%$ ). Nevertheless, even the lowest loading of ADEPAE comonomeric units led to the reduction of $\mathrm{HRC}$ values from $729(\mathrm{~J} / \mathrm{g} \cdot \mathrm{K})$ for PS to $541(\mathrm{~J} / \mathrm{g} \cdot \mathrm{K})$ for poly(S-co-ADEPAE). While this result is quite encouraging, it cannot be easily explained (a normalized value of 2705 is not plotted in Figure 8).

Table 7. PCFC parameters for S-based polymers.

\begin{tabular}{cccccc}
\hline Parameter & PS & Poly(S-co-DEAMP) & Poly(S-co-DEAEP) & Poly(S-co-DEMEP) & Poly(S-co-ADEPAE) \\
\hline$[\mathrm{M}] /[\mathrm{co}-\mathrm{M}]$ & 0 & $0.880 / 0.120$ & $0.902 / 0.098$ & $0.873 / 0.127$ & $0.992 / 0.008$ \\
$($ mole fraction) & & & & & \\
P content $(\mathrm{wt} \%)$ & 0 & 3.1 & 2.6 & 3.2 & 0.2 \\
$T_{\text {PHRR }}\left({ }^{\circ} \mathrm{C}\right)$ & 420 & 383 & 392 & 419 & 423 \\
PHRR $(\mathrm{W} / \mathrm{g})$ & 703 & 512 & 557 & 389 & 499 \\
PHRR $(\%$ reduction) & 0 & 27 & 21 & 45 & 29 \\
THR (kJ/g) & 38.5 & 33.2 & 33.1 & 33.6 & 50.1 \\
HRC $(\mathrm{J} / \mathrm{g} \cdot \mathrm{K})$ & 729 & 561 & 609 & 423 & 1.0 \\
PCFC char yield & 1.1 & 3.1 & 5.2 & 3.8 & \\
(wt. \%) & & & &
\end{tabular}




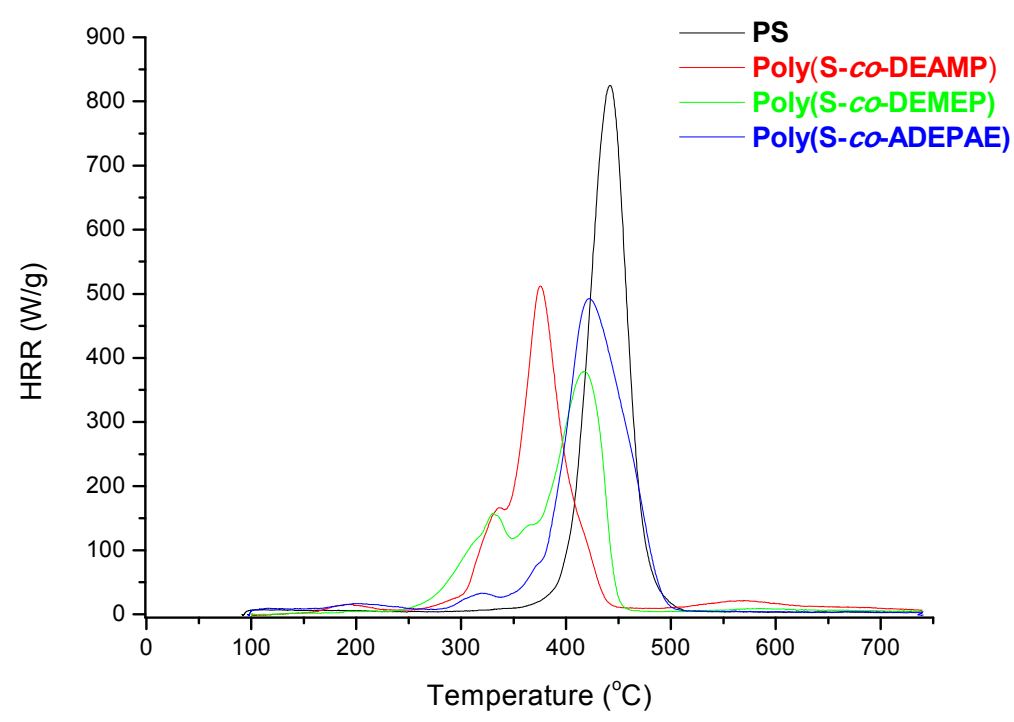

Figure 7. Plots of HRR versus temperature for PS, poly(S-co-DEAMP), poly(S-co-DEMEP) and poly(S-co-ADEPAE).

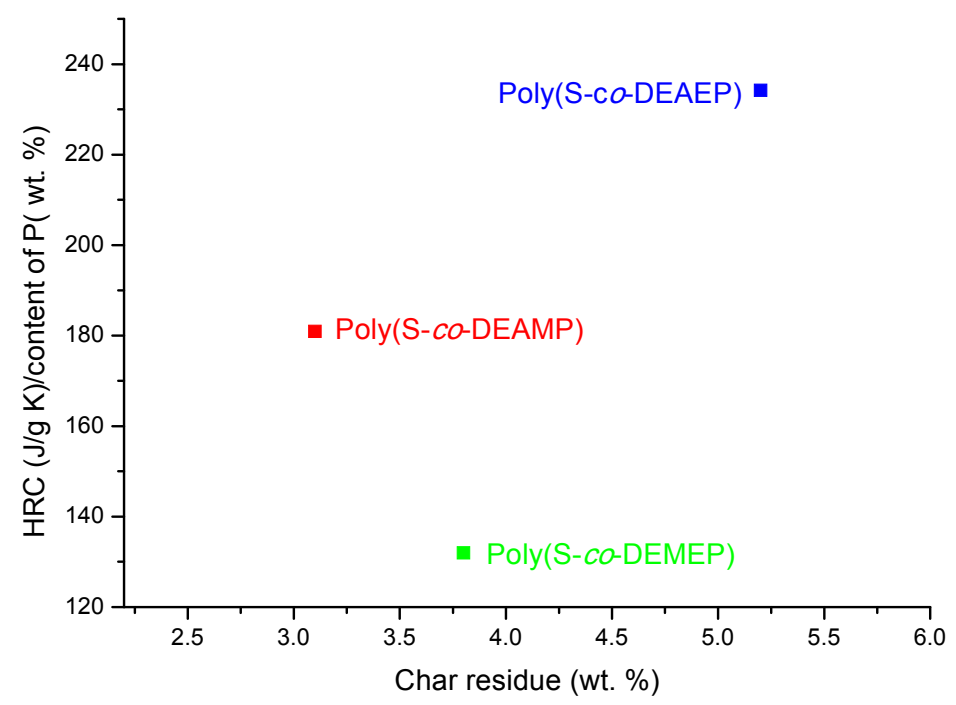

Figure 8. Ratio of HRC to the content of P versus PCFC char residue for the copolymers of S.

The mechanistic aspects of flame retardation in MMA- and S-based systems ought to be different to that of PAN as the primary modes of degradation of both PS and PMMA differ from that of PAN owing to the differences in their chemical constitution. In any case, the initial point of degradation is believed to be activated by the modifying groups containing only $\mathrm{P}$ atoms or both $\mathrm{P}$ and $\mathrm{N}$ atoms. As is shown previously, in the case of MMA-based polymers trans-esterification route is highly probable leading to a formation of char precursors $[8,11,12]$, whilst in the case of PS the elements of mechanism are less obvious [11,13].

\section{Conclusions}

The main conclusions from the present study can be summarised as follows:

(1) The synthetic routes to the $\mathrm{P}$ - and $\mathrm{P} / \mathrm{N}$-containing monomers and the preparative procedures for various polymeric materials were found to be quite facile and without significant unwanted side 
reactions, and resulting in appreciable yields. The thermo-gravimetric analyses, in different atmospheres, showed earlier cracking of the pendant modifying groups quite earlier on in all cases (data not reported here). It is to be inferred here that the modifying groups aid in changing the degradative behaviours of the polymeric matrices, at early stages, thus initiating the flame retarding effects in these systems. The actual physiochemical pathways underlying these elements are more or less well established in the case of PAN and PMMA, but are far from clear in the case of PS $[7,12,13]$.

(2) Pyrolysis Combustion Flow Calorimetry (PFCF) has been found to be a very useful screening technique, especially, in establishing the efficacies of the different modifying groups towards flame retarding some base polymeric materials. For typical char forming polymers, like PAN, there exist stronger correlations between the parameters obtained through PCFC measurements and the char residues from TGA runs, heats of combustion values obtained from oxygen bomb calorimetry, indices from the LOI measurements, etc. [10]. Values of the HRC values normalised to the $\mathrm{P}$ contents $(\mathrm{wt} \%)$ can be considered as useful tool in ranking the various P-containing modifying groups in terms of their efficacies to flame-retard non-halogenated chain-growth polymers considered in the present work.

\section{Acknowledgments}

The authors wish to thank the Engineering and Physical Sciences Research Council (EPSRC), UK, for the financial support and for the provision of a Research Associateship (grant number: EP/F068867/1).

\section{Author Contributions}

Dr. Svetlana Tretsiakova-McNally (S.T.-M.) was the researcher in the project (EPSRC: EP/F068867/1), and she undertook the main body of the experimental work. She also substantially contributed towards the manuscript; Dr. Paul Joseph (P.J.) was the Principal Investigator of the project, and has directly supervised S.T.-M. during the entire programme. He also edited the manuscript.

\section{Conflicts of Interest}

The authors declare no conflict of interest.

\section{References}

1. Cogen, J.M.; Lin, T.D.; Lyon, R.E. Correlations between pyrolysis combustion flow calorimetry and conventional flammability tests with halogen-free flame retardant polyolefin compounds. Fire Mater. 2009, 33, 33-50.

2. Sonnier, R.; Vahabi, H.; Ferry, L.; Lopez-Cuesta, J.M. Pyrolysis-combustion flow calorimetry: A powerful tool to evaluate the flame retardancy of polymers. In Fire and Polymers VI: New Advances in Flame Retardant Chemistry and Science; Morgan, A.B., Wilkie, C.A., Nelson, G.L., Eds.; ACS Symposium Series 118; American Chemical Society: Washington, DC, USA, 2012; pp. 361-390. 
3. Lyon, R.E.; Walters, R.N. Pyrolysis combustion flow calorimetry. J. Anal. Appl. Pyrolysis 2004, $71,27-46$.

4. Armarego, W.L.F.; Chai, C.L. Purification of Laboratory Chemicals, 5th ed.; Elsevier Science: Cornwall, UK, 2003.

5. Liepins, R.; Surles, J.R.; Morosoff, N.; Stannett, V.; Duffy, J.J.; Day, F.H. Localized radiation grafting of flame retardants to polyethylene terephthalate. II. Vinyl phosphonates. J. Appl. Polym. Sci. 1978, 22, 2403-2414.

6. Nair, C.P.R.; Clouet, G.; Brossas, J. Copolymerization of diethyl-2-(methacryloyloxy)ethyl phosphate with alkyl acrylates: Reactivity ratios and glass transition temperatures. J. Appl. Polym. Sci. A 1988, 26, 1791-1807.

7. Ebdon, J.R.; Hunt, B.J.; Joseph, P.; Wilkie, T.K. Flame retardance of polyacrylonitriles covalently modified with phosphorus- and nitrogen containing groups. In Fire Retardancy of Polymers: New Strategies and Mechanisms; Hull, T.R., Kandola, B.K., Eds.; Royal Society of Chemistry: Cambridge, UK, 2009; pp. 331-364.

8. Ebdon, J.R.; Hunt, B.J.; Joseph, P.; Konkel, C.S.; Price, D.; Pyrah, K.; Hull, T.R.; Milnes, G.J.; Hill, S.B.; Lindsay, C.I.; et al. Thermal degradation and flame retardance in copolymers of methyl methacrylate with diethyl(methacryloyloxymethyl)phosphonate. Polym. Degrad. Stab. 2000, 70, 425-436.

9. Ebdon, J.R.; Price, D.; Hunt, B.J.; Joseph, P.; Gao, F.; Milnes, G.J.; Cunliffe, L.K. Flame retardance in some polystyrenes and poly(methyl methacrylate)s with covalently bound phosphorus-containing groups: Initial screening experiments and some laser pyrolysis mechanistic studies. Polym. Degrad. Stab. 2000, 69, 267-277.

10. Joseph, P.; Tretsiakova-McNally, S. Combustion behaviours of chemically modified polyacrylonitrile polymers containing phosphorylamino groups. Polym. Degrad. Stab. 2012, 97, 2531-2535.

11. Joseph, P.; Tretsiakova-McNally, S. Mechanistic aspects of flame retardation by phosphorus-containing groups in some chain growth polymers. In Fire and Polymers VI: New Advances in Flame Retardant Chemistry and Science; Morgan, A.B., Wilkie, C.A., Nelson, G.L., Eds.; ACS Symposium Series 118; American Chemical Society: Washington, DC, USA, 2012; pp. 37-50.

12. Price, D.; Cunliffe, L.K.; Bullte, K.J.; Hull, T.R.; Milnes, G.J.; Ebdon, J.R.; Hunt, B.J.; Joseph, P. Thermal behaviour of covalently bounded phosphonate flame-retarded poly(methyl methacrylate) systems. Polym. Adv. Technol. 2008, 19, 710-723.

13. Price, D.; Cunliffe, L.K.; Bullte, K.J.; Hull, T.R.; Milnes, G.J.; Ebdon, J.R.; Hunt, B.J.; Joseph, P. Thermal behaviour of covalently bonded phosphate and phosphonate flame retarded polystyrene systems. Polym. Degrad. Stab. 2007, 92, 1101-1114.

(C) 2015 by the authors; licensee MDPI, Basel, Switzerland. This article is an open access article distributed under the terms and conditions of the Creative Commons Attribution license (http://creativecommons.org/licenses/by/4.0/). 